\title{
Ventilated cloud cavitating flow around a blunt body close to the free surface
}

\author{
Yiwei Wang, Chang Xu, Xiaocui Wu, Chenguang Huang, ${ }^{*}$ and Xianqian Wu \\ Key Laboratory for Mechanics in Fluid Solid Coupling Systems, Institute of Mechanics, \\ Chinese Academy of Sciences, Beijing 100190, China \\ and School of Engineering Science, University of Chinese Academy of Sciences, Beijing 100049, China
}

(Received 4 September 2016; published 22 August 2017)

\begin{abstract}
Ventilated cavitation occurs as a complicated problem if the free surface is close to the cavity boundary around a high-speed underwater vehicle. The present study investigate the cavitating flow around a blunt axisymmetric body very near the free surface. A typical experiment is conducted by using a launching system with a split Hopkinson pressure bar device, and a numerical scheme is established based on large eddy simulation and volume-of-fluid methods. Unsteady behavior, including air entrainment and shedding of the cloud cavity, is observed, and high consistency is achieved between numerical and experimental results. Distinctions of evolution features between the cavities on the upper and lower sides are presented and analyzed. First, strong entrainment of noncondensable air occurs and changes the fluid property inside the cavity, which makes the cavity larger. Moreover, given the small distance between the vehicle and the free surface, the re-entrant jet generated in the upper part is thin and cannot completely cut off the main cavity, which causes the upper part of the cavity to remain approximately unchanged after the growth stage without the occurrence of the shedding phenomenon. Finally, the evolution of vortex structures is also discussed by comparisons with the motions of the air entrainment, re-entrant jet, and shedding cavity.
\end{abstract}

DOI: 10.1103/PhysRevFluids.2.084303

\section{INTRODUCTION}

The interaction between the cavitation region and the free surface is essential to the fast cruising of surface vehicles. Ventilated cavitation occurs as an extremely complicated problem if the free surface is close to the vehicle or the cavity boundary mentioned by Faltinsen and Semenov [1]. The gas above the free surface can be entrained into the cavity, which may change the dynamic behavior of the cavity. Furthermore, the effect of wave elevation is coupled with the distribution of the cavitation region.

Relevant studies on the interactions between the free surface and the cavitating flow are limited. Most relevant studies have focused on stable cavitation, and the interfaces of the cavity and the free surface are separated from each other. Early experimental results were obtained by Dawson and Bate [2] in a free-surface water tunnel under low flow speed conditions. They used artificial ventilation to generate stable supercavitating flows around a wedge-shape hydrofoil. Theoretical analysis and numerical simulation are primarily adopted at present. Early research based on linearized theory can be found in the book of Franc and Michel [3]. Faltinsen and Semenov [1] established a numerical approach within a unified framework on the supercavitating flow near free surface. They discussed the effects of submerged depth, Froude number, cavitation number, and other parameters on the cavitation shape and lift force. Bal et al. presented a boundary element method [4-6] for cavitating flows around hydrofoils near free surface, including surface-piercing cavitating cases. The effect of free surface was also considered in several studies on supercavitating flow in shallow water $[7,8]$. Wang et al. [9] established experimental and numerical results to analyze the unsteady cavitating

\footnotetext{
*Corresponding author: huangcg@imech.ac.cn
} 


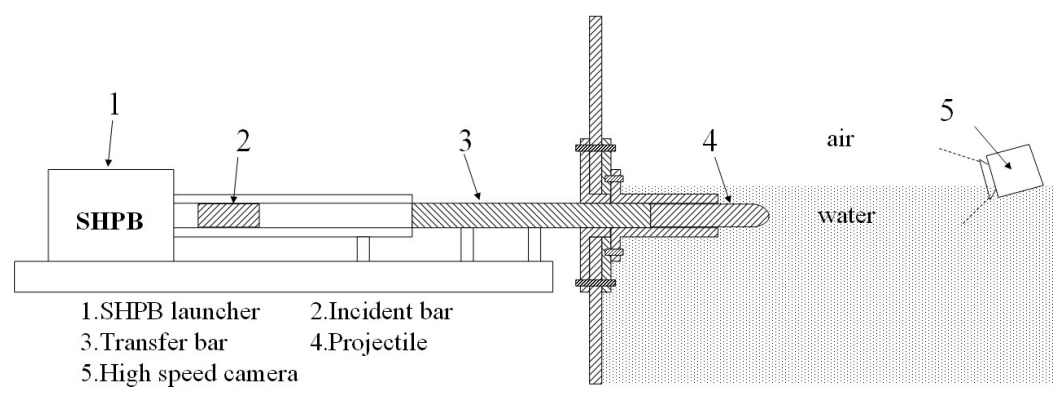

FIG. 1. Components of the underwater launch system.

flow around an axisymmetric projectile near a free surface. The effects of the free surface on unsteady behavior were investigated based on the results under a typical condition.

Moreover, neglecting the influence of the free surface, the dynamic characteristics of the cloud cavitating flow are often linked with the motion of vortices. The evolution of cavities, including their growth, re-entrant, shedding, and collapse were investigated based on the experimental [10-12] and numerical [13-15] approaches. For the numerical approaches, turbulence-solving approaches were emphasized. Modified Reynolds-averaged Navier-Stokes equations (RANS) [16-21] and large eddy simulation (LES) [22-28] methods are common methods of study.

Ventilation, as an important control method for cavitating flow, is usually investigated under artificial conditions. Except for drag reduction [29], ventilation of noncondensable gas can also weaken the instability of the cavity or eliminate the collapse of the unstable cavity. However, only a few studies focused on partial cavitation. Pham et al. [30] performed air injection through a slit in the vicinity of the leading edge on a foil. Their results validated the effectiveness of decreasing the amplitude of instabilities. Wang et al. [31] transformed the shedding phenomenon of an entire cavity into partial shedding at the closure of the cavity by passive ventilation around a projectile. Young and Harwood examined the ventilated cavities on a surface-piercing hydrofoil at moderate Froude numbers [32,33]. The formation and elimination mechanisms are proposed and stability regions of the different flow regimes are plotted. In addition, the authors also give a comprehensive review of scaling effect on the ventilation of lifting bodies.

The aforementioned literature analysis shows that research on unstable cavitation near the free surface is still difficult and inadequate; studies on unsteady cavitating flow near the free surface involving surface pierce are very rare. Many complicated phenomena and relative mechanisms should be revealed. In the present study, we investigated the cavitating flow around a blunt axisymmetric body close to a free surface. New phenomena involving interactions between cavitation and local air entrainment were observed and analyzed. A typical experiment is conducted by using a launching system with a split Hopkinson pressure bar (SHPB) device, and a numerical method is established based on the LES and volume-of-fluid (VOF) methods. The distinctions of evolution features between the cavities on the upper and lower sides are presented. The effects of the free surface and the gas entrainment on cavity evolution are investigated.

\section{EXPERIMENTAL SETUP}

The experiment is performed by using a launching system (as shown in Fig. 1) based on SHPB technology [34], which can transiently accelerate a projectile with only a slight disturbance on the water. There is a transfer bar shown in the figure with one side out of the water, while the other side is inside. When the incident bar impacts the transfer bar, a strong stress wave is generated and propagates into the projectile, which can accelerate the projectile very quickly. So the total system has very little disturbance to the flow field before the launching process. The projectile used in this study has a blunt and axisymmetric shape. The total length is $150 \mathrm{~mm}$, and the diameter is $37 \mathrm{~mm}$. 


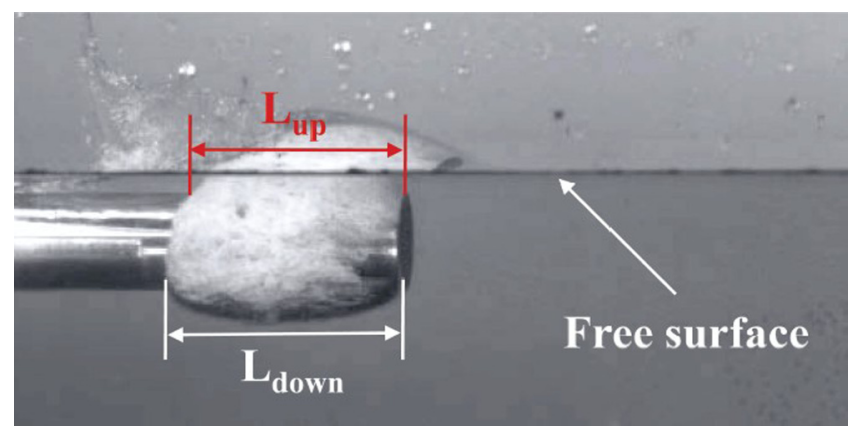

FIG. 2. Typical cavitation photograph. The cavity length on the upper and lower side of the projectile is marked by $L_{\text {up }}$ and $L_{\text {down }}$. The free surface is pointed out by an arrow.

Photographs of typical cavitation are obtained using a high-speed camera with a sampling frequency of 12000 frames per second. The cavity is non-asymmetrically affected by the free surface. Thus, the lengths on the upper and lower sides of the cavity are measured by pixel analysis (as shown in Fig. 2). The precision of the cavity length is approximately a pixel of the image, which amounts to approximately $0.65 \mathrm{~mm}$. For the typical condition investigated in the present study, the distance between the free surface and the upper side of the projectile is $5 \mathrm{~mm}$, and the analysis of the obtained images indicates that the speed is approximately uniform at $19.1 \mathrm{~m} / \mathrm{s}$. The cavitation number is calculated as 0.537 .

\section{NUMERICAL METHODS}

\section{A. Governing equations}

To simulate the motions of liquid water and vapor and air, including the phase change, mixture and multiphase flow equations are adopted. Continuity and momentum equations for the mixture are established as follows.

$$
\begin{gathered}
\frac{\partial \rho}{\partial t}+\frac{\partial\left(\rho u_{j}\right)}{\partial x_{j}}=0 \\
\frac{\partial\left(\rho u_{i}\right)}{\partial t}+\frac{\partial\left(\rho u_{i} u_{j}\right)}{\partial x_{j}}=-\frac{\partial p}{\partial x_{j}}+\frac{\partial}{\partial x_{j}}\left(\mu \frac{\partial u_{j}}{\partial x_{j}}\right),
\end{gathered}
$$

where $u_{i}$ is the velocity component in the $i$ direction, and $p$ is the pressure.

Laminar viscosity $\mu$ is defined as a volume-weighted average of the three components as

$$
\mu=\left(1-\alpha_{v}-\alpha_{a}\right) \mu_{l}+\alpha_{v} \mu_{v}+\alpha_{a} \mu_{a}
$$

where $\alpha_{v}$ and $\alpha_{a}$ are the vapor and air volume fractions, respectively.

Mixture density $\rho$ is defined as

$$
\rho=\left(1-\alpha_{v}-\alpha_{a}\right) \rho_{l}+\alpha_{v} \rho_{v}+\alpha_{a} \rho_{a} .
$$

The volume fractions of vapor and air are governed by the following mass transfer equations:

$$
\begin{gathered}
\frac{\partial\left(\alpha_{v} \rho_{v}\right)}{\partial t}+\frac{\partial\left(\alpha_{v} \rho_{v} u_{j}\right)}{\partial x_{j}}=\dot{m}^{+}-\dot{m}^{-} \\
\frac{\partial\left(\alpha_{a} \rho_{a}\right)}{\partial t}+\frac{\partial\left(\alpha_{a} \rho_{a} u_{j}\right)}{\partial x_{j}}=0 .
\end{gathered}
$$


The source terms $\dot{m}^{+}$and $\dot{m}^{-}$in the vapor transport equation (5) represent the mass transfer rates of evaporation and condensation, which are derived from the bubble dynamics equation of the generalized Rayleigh Plesset equation by Zwart et al. [35] as follows:

$$
\begin{gathered}
\dot{m}^{+}=F_{\text {vap }} \frac{3 a_{\text {nuc }}\left(1-\alpha_{v}\right) \rho_{v}}{R_{B}} \sqrt{\frac{2}{3} \frac{\max \left(p_{v}-p, 0\right)}{\rho_{l}}}, \\
\dot{m}^{-}=F_{\text {cond }} \frac{3 \alpha_{v} \rho_{v}}{R_{B}} \sqrt{\frac{2}{3} \frac{\max \left(p-p_{v}, 0\right)}{\rho_{l}}} .
\end{gathered}
$$

In Eqs. (7) and (8) generalized bubble radius $R_{B}$ is set at $10^{-6} \mathrm{~m}$, nucleation site volume fraction $a_{\text {nuc }}$ is set at $5 \times 10^{-4}$, the evaporation coefficient is set at 50 , and the condensation coefficient is set at 0.01 . The chosen parameter values are based on the work of Zwart et al. The parameters have been discussed and found to work well for a variety of fluids and devices. The parameters and the recommended value of the cavitation model are widely used [9]. In addition, some studies have shown that within a certain range, the parameters have little effect on the results of cloud cavitating flow [36].

\section{B. LES approach}

The governing equations are solved by a LES approach with a Smagorinsky-Lilly subgrid scale (SGS) model. Applying a Favre-filtering operation to Eqs. (1) and (2) derives the following LES equations:

$$
\begin{gathered}
\frac{\partial \rho}{\partial t}+\frac{\partial\left(\rho \bar{u}_{j}\right)}{\partial x_{j}}=0 \\
\frac{\partial\left(\rho \bar{u}_{i}\right)}{\partial t}+\frac{\partial\left(\rho \bar{u}_{i} \bar{u}_{j}\right)}{\partial x_{j}}=-\frac{\partial \bar{p}}{\partial x_{j}}+\frac{\partial}{\partial x_{j}}\left(\mu \frac{\partial \bar{u}_{j}}{\partial x_{j}}\right)-\frac{\partial \tau_{i j}}{\partial x_{j}},
\end{gathered}
$$

where the overbars denote filtered quantities. The SGS stress $\tau_{i j}$ as the extra term in Eq. (10), which must be modeled, is defined as follows:

$$
\tau_{i j}=\rho\left(\overline{u_{i} u_{j}}-\bar{u}_{i} \bar{u}_{j}\right)
$$

The Boussinesq hypothesis is employed, in which the SGS stress is computed from

$$
\tau_{i j}-\frac{1}{3} \tau_{k k} \delta_{i j}=-2 \mu_{t} \bar{S}_{i j}
$$

where $\mu_{t}$ is the SGS turbulent viscosity. Isotropic part $k k$ is not modeled but added to the filtered static pressure term. $S_{i j}$ is the rate-of-strain tensor for the resolved scale defined by

$$
\bar{S}_{i j} \equiv \frac{1}{2}\left(\frac{\partial \bar{u}_{i}}{\partial x_{j}}+\frac{\partial \bar{u}_{j}}{\partial x_{i}}\right) .
$$

The SGS turbulent viscosity $\mu_{t}$ is closed by the Smagorinsky-Lilly model, and calculated by $\mu_{t}=\rho L^{2}{ }_{s} \sqrt{2 \bar{S}_{i j} \bar{S}_{i j}}$, where $L_{s}$ is the mixing length for subgrid scales. It is computed by $L_{s}=$ $\min \left(\kappa d, C_{s} V^{1 / 3}\right)$, where $\kappa$ is the von Karman constant, $d$ is the distance to the closet wall, $C_{s}$ is the Smagorinsky constant set as 0.1 , and $V$ is the volume of the computational cell.

\section{Simulation procedure}

Unsteady numerical simulations are performed based on the finite volume method with coupled scheme by using the commercial computational fluid dynamics software ANSYS-FLUENT. The equations are discretized by a second-order implicit scheme in time and a bounded central differencing scheme in space. An implicit scheme is also used for time discretization of the volume 


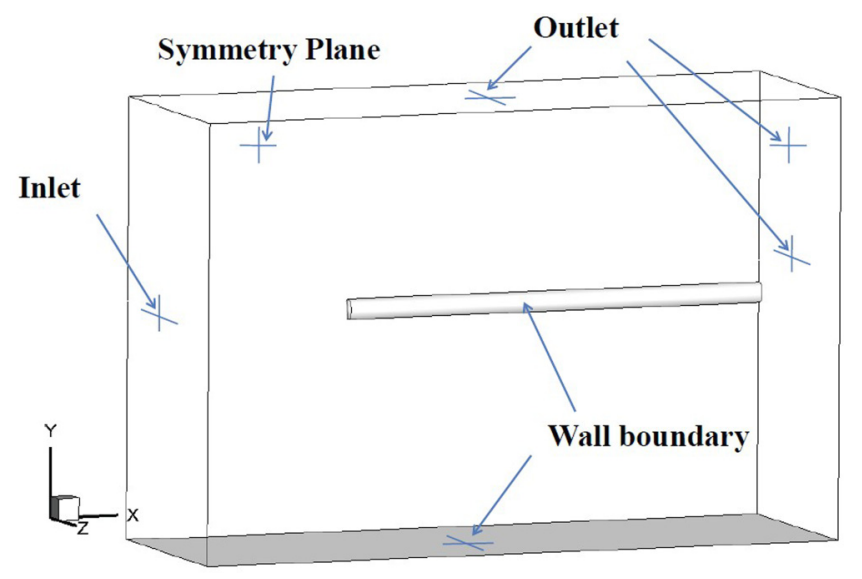

FIG. 3. Calculated domain and boundary conditions in the simulation. The defined velocity-inlet, pressureoutlet, and wall boundary conditions are marked.

fraction equation which is compatible with the cavitation model. The pressure staggering option is selected for pressure interpolation. The modified high-resolution interface-capturing scheme is used for the volume fraction, which is more robust than the explicit geometric reconstruction scheme. The unsteady cavitating flow simulations are started from a uniform flow field because the whole acceleration process is very short. The time step is set as $10^{-5} \mathrm{~s}$.

The computational domain is discretized with a block-structured grid, which is refined around the model and near the free surface, and only half of the model is considered (as shown in Fig. 3). A semi-infinite projectile model is used, and the effect of the tail on the shoulder cavity is neglected. The cell number is approximately $4 \times 10^{6}$ with good orthogonality (as shown in Fig. 4). The model is fixed, with the free surface moving toward the model. The inlet velocity is set as $19.1 \mathrm{~m} / \mathrm{s}$, with no turbulent perturbations for the velocity-inlet boundary condition. Independence analysis and validation of a similar grid on the simulation of the typical evolution of cloud cavitating flow can be found in the reference paper of Wang et al. [9].

To verify the stability of the original mesh size for the simulation, we produce a new refined mesh that contains about $3 \times 10^{7}$ cells using the same mesh method. The resultant cavity profiles are compared between that in the previous simulation and that in the experimental results. Figure 5

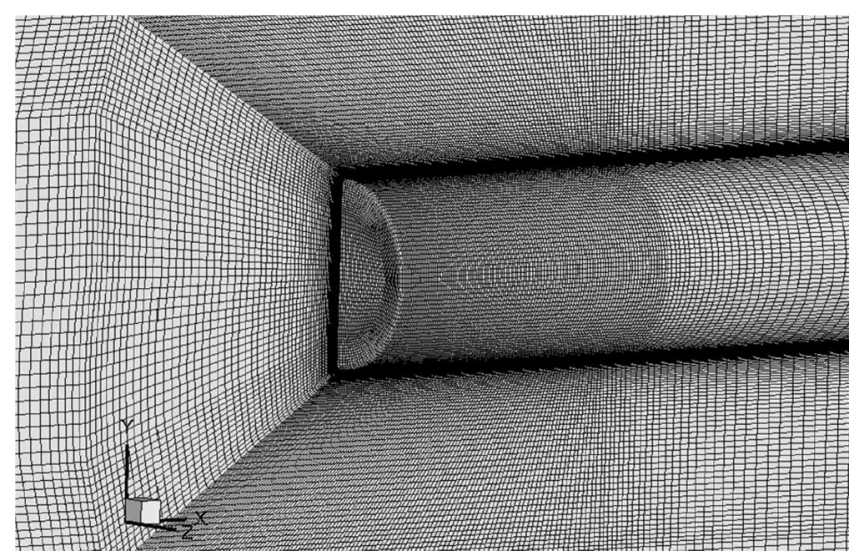

FIG. 4. Mesh near the head of the projectile. 


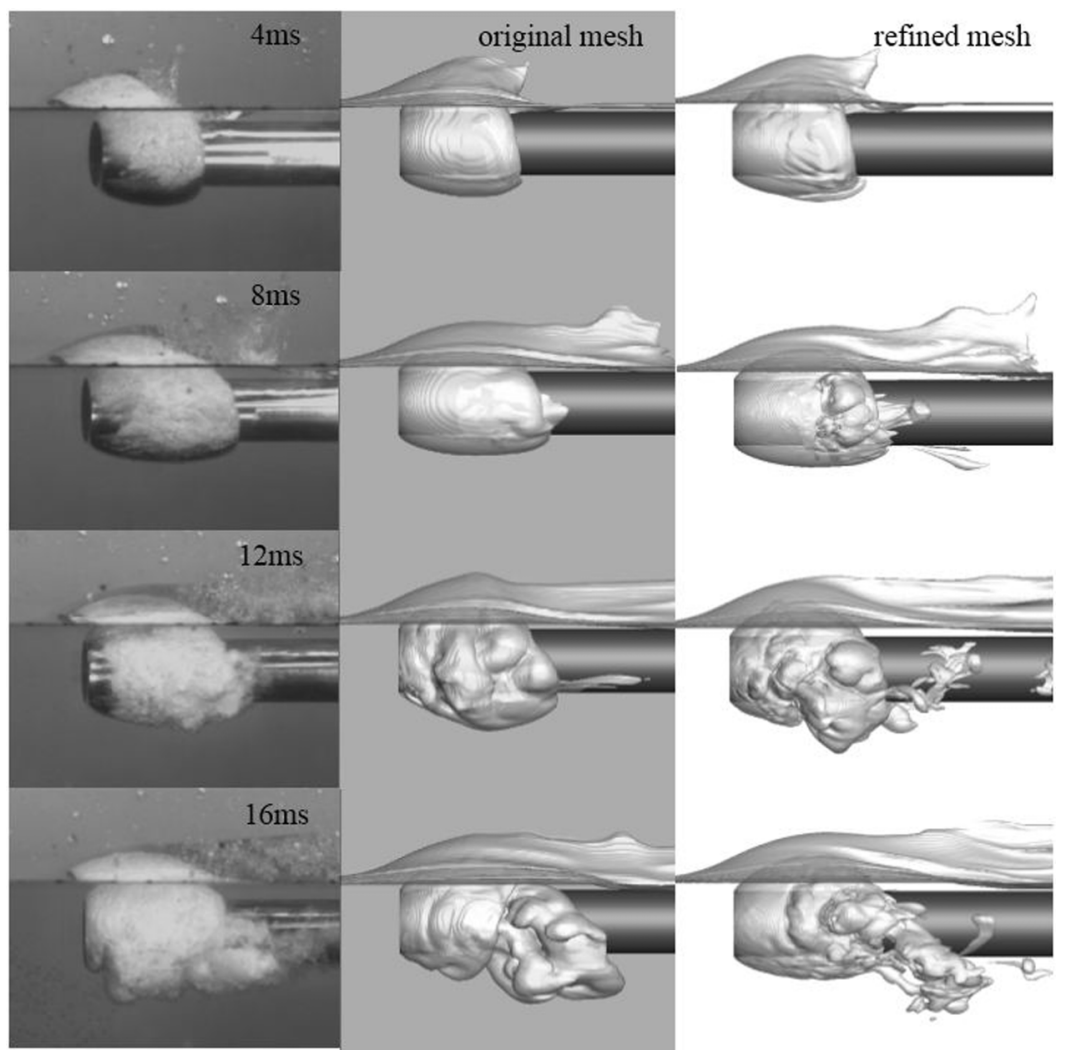

FIG. 5. Comparison of the cavity profiles between the experimental figures and the simulated results of the original mesh and refined mesh.

shows the results. In the present study, we consider the main features of cavity evolution to a greater extent than in other attributes. By comparing the images shown in Fig. 5, we find that the refined mesh simulation results are consistent with the original results of cavity evolution. The specific features for comparison include cavity length, re-entrant jet fronts, and cavity shape.

After verifying the mesh independence of the simulation method, the simulation results of the original mesh are used for further analysis and discussion. The results show that the mesh plan is fairly stable. The simulated results of small cell sizes remain fine.

\section{RESULTS AND DISCUSSION}

\section{A. Overall evolution of cavities}

The quasiperiodic development of cavity shape is obtained from the experimental and numerical results. The variations of cavity lengths on the upper and lower sides are shown in Fig. 6. The length development and variation of the obtained numerical results are fairly consistent with those of the experiments, including the phenomenon of the breaking lower side cavity and the relationship between cavities on the upper and lower sides. The results of a submerged case with the same cavitation number are also provided for comparison. The free surface is $40 \mathrm{~mm}$ away from the projectile; thus, the effect of the free surface is negligible.

The cavity shapes in experiment photographs and numerical results at typical moments of different stages are as shown in Figs. 7-9. Cavities in the stage of cavity growth and air entrainment are shown in Fig. 7. The cavities incept when the projectile moves suddenly, and the free surface is elevated at 


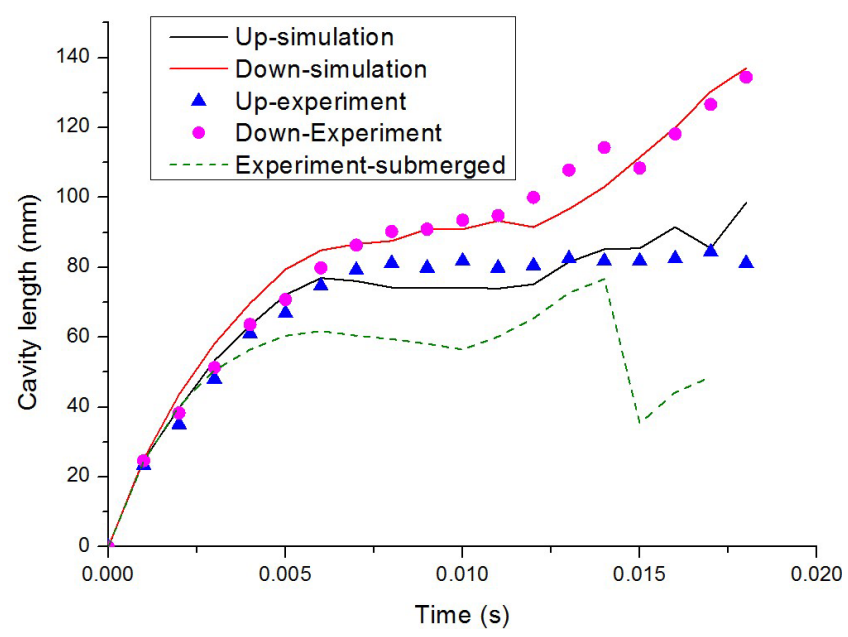

FIG. 6. The comparison of the variation of cavity length between numerical and experimental results.

the same time. The cavities appear transparent at the beginning. Then, at the moment of $2 \mathrm{~ms}$, the two interfaces of the cavity boundary and free surface intersect with each other. A hole is generated, and air entrainment emerges from the open air into the cavity. A foamlike pattern caused by the strong immersion appears in the experiment photograph, whereas the blue surface also separates from the translucent surface in the numerical results. Subsequently, a sharp angle facing downstream on the free surface is induced by the stream of the air entrainment at the moments of 3 and $4 \mathrm{~ms}$. The cavity on the upper side is shorter and thicker than that on the lower side.

The re-entrant jet is generated and has an important role in the unsteadiness from 5 to $14 \mathrm{~ms}$. This stage can be divided into two parts. The cavity boundary is approximately smooth before $8 \mathrm{~ms}$ (as shown in Fig. 8). Cavity growth is stable, and the cavity on the lower side is always longer than that on the upper side. Water layer breaking induced by the air entrainment is clearly shown in the experiment photographs. Meanwhile, the large deformation of the free surface caused by the initial impact is shown in the numerical results.

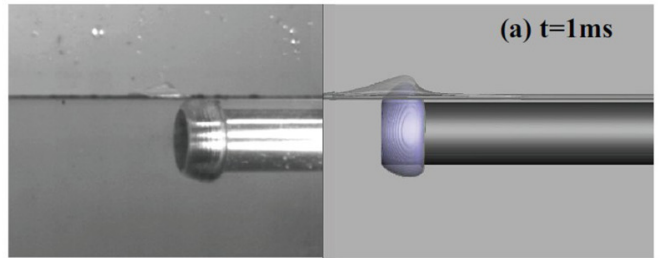

(b) $\mathrm{t}=\mathbf{2} \mathrm{ms}$
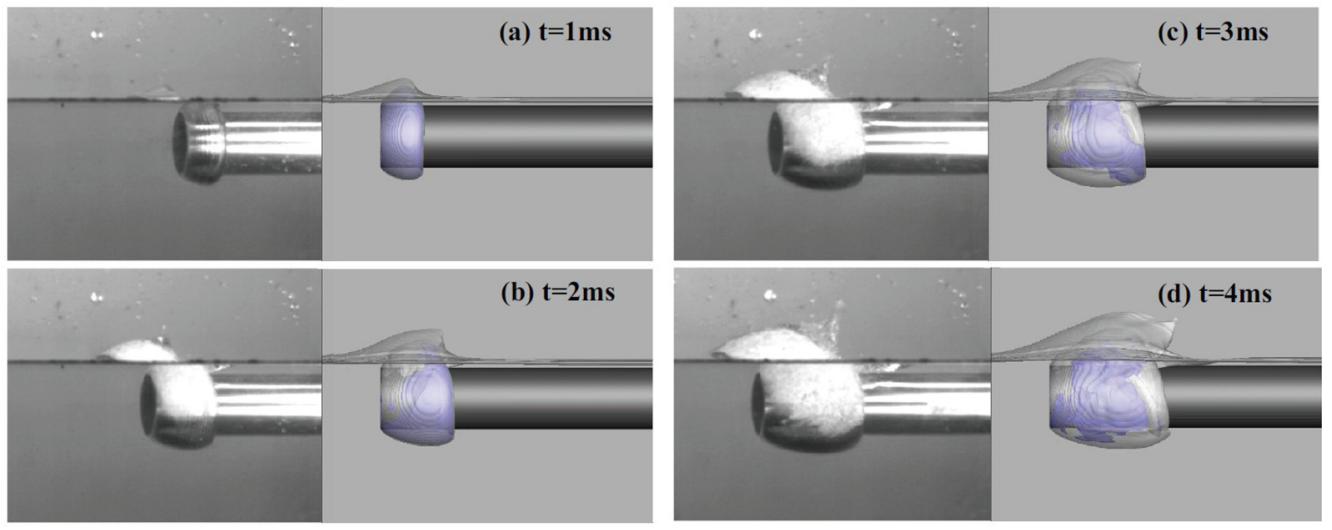

FIG. 7. Time evolution of cavitation patterns obtained from the experiment and simulation in the stage of cavity growth and air entrainment. The translucent isosurface represents where the volume fraction of liquid water is 0.9 , while the blue isosurface represents where the volume fraction of vapor is 0.5 , which can demonstrate the front of the air entrainment. 

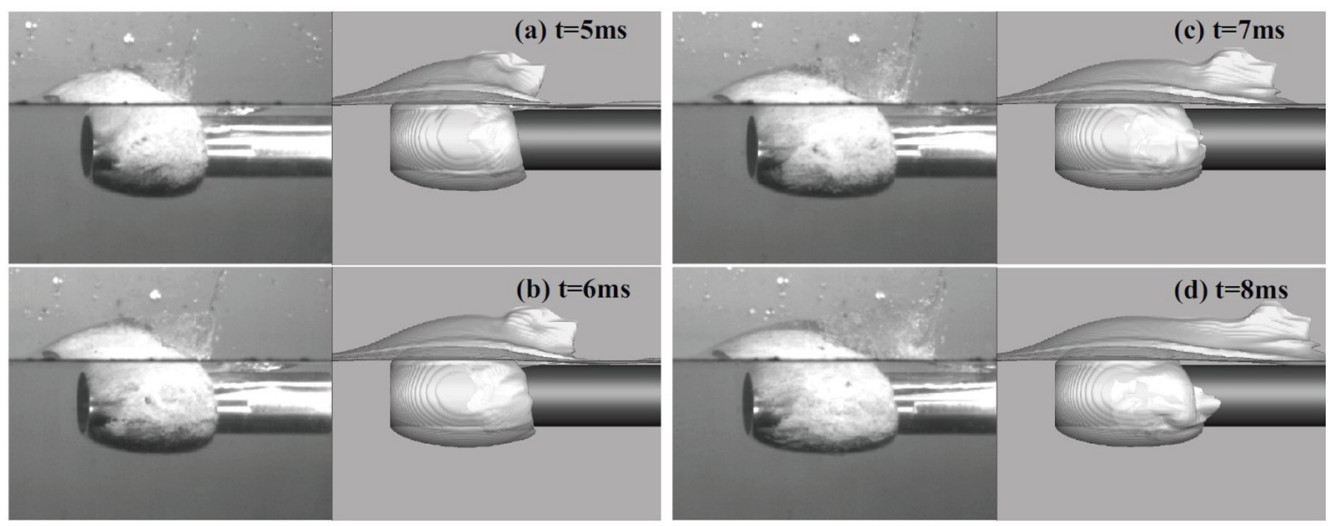

FIG. 8. Time evolution of cavitation patterns obtained from the experiment and simulation in the earlier part of the re-entrant jet action stage. The translucent isosurface represents where the volume fraction of liquid water is 0.9 .

Obvious disturbance inside the cavity caused by the nearby free surface and the air injection is present in Fig. 9. Initially the boundary of the cavity becomes unsmooth near the cavity closure [as shown in Figs. 9(a)-9(c)]. Then, the bumps inside the cavity become larger and extend to the lower region of the cavity [as shown in Figs. 9(d)-9(f)]. In the experimental figures, the disturbance propagates along the white foamlike structure, which is a typical feature of the re-entrant jet. Gas leakages appear in the experimental and numerical results near the lower region of the cavity closure [pointed out by red arrows in Fig. 9(d)].

Large clouds shed from the lower part of the main cavity along with gas leakage from 15 to $18 \mathrm{~ms}$. The large clouds appear in the experimental and numerical results (Fig. 9). The shedding cavities
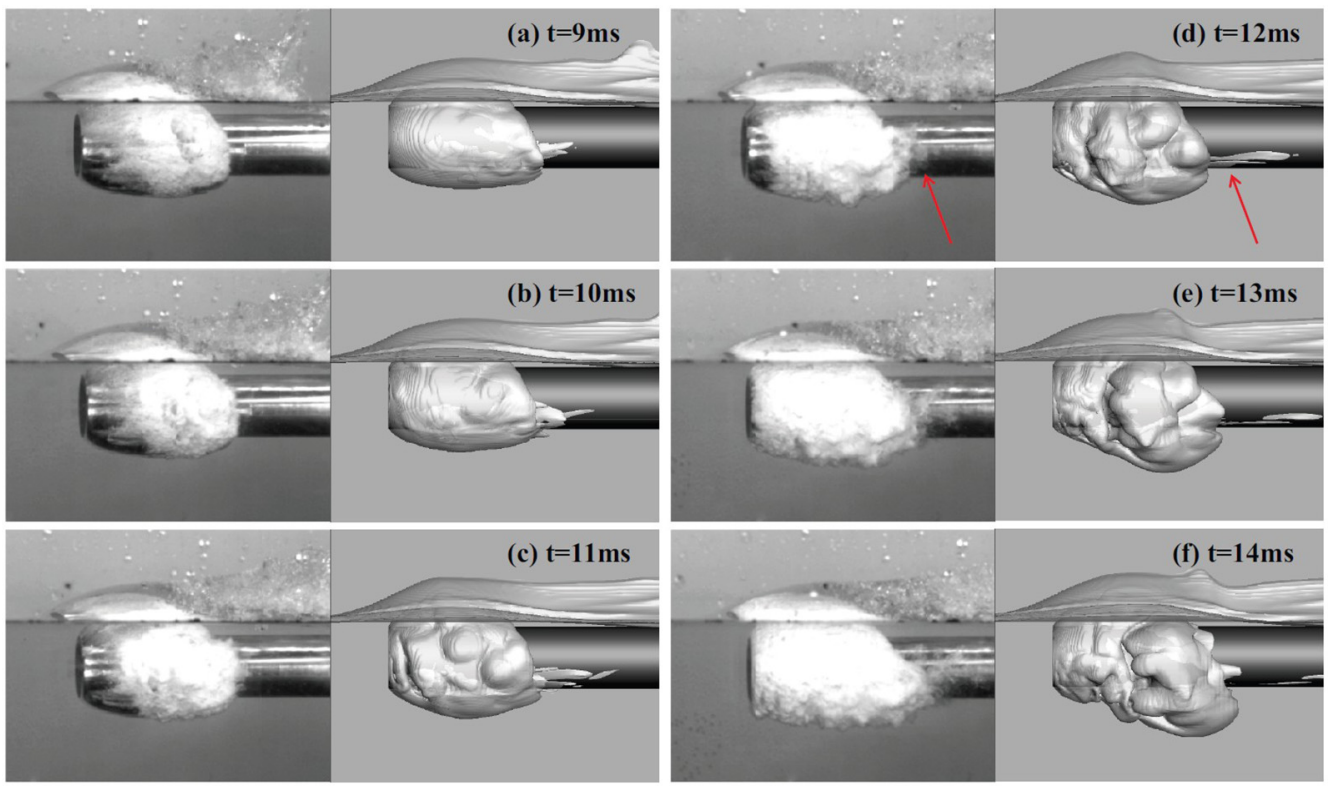

FIG. 9. Time evolution of cavitation patterns obtained from the experiment and simulation in the later part of the re-entrant jet action stage. The translucent isosurface represents where the volume fraction of liquid water is 0.9 . 

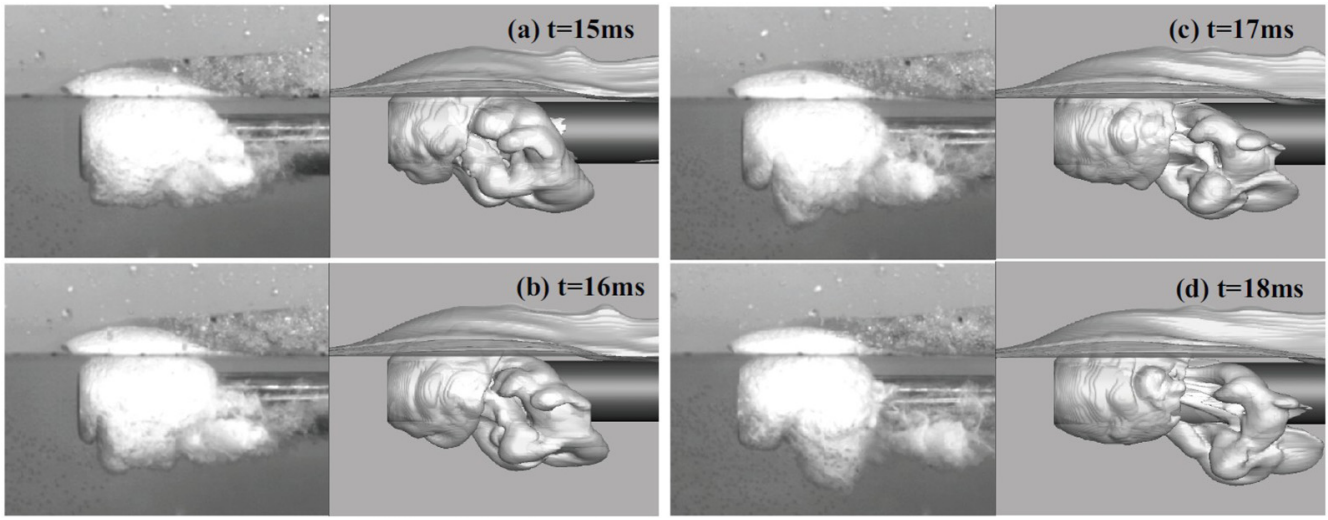

FIG. 10. Time evolution of cavitation patterns obtained from the experiment and simulation in the cavity shedding stage. The translucent isosurface represents where the volume fraction of liquid water is 0.9.

transform from slug bubbles into strips. However, a large gap is not observed in the numerical results, and the gap is considered as the trailing edge of the cavity with which we calculate the length in Fig. 6. Thus, a notable difference in length between the numerical and experimental results is observed. This may be due to the decrease of the projectile speed in the water tank experiment and the changes of the trajectory during the experiment. Because the projectile pitches downward after long-range movement in experiments, large-scale cavity shedding occurs more easily near the shoulder on the lee side (lower side), which does not appear in numerical results. Notably, the cavity stays stable on the upper side of the projectile in the numerical and experimental results without visible shedding (Fig. 10).

Compared with the submerged case as shown in Fig. 6, the quasiperiodical evolution of the cavity exhibits typical cavity patterns, as shown in Fig. 11. We can see the development, including the cavity growth [in Figs. 11(a) and 11(b)], re-entrant jet [in Figs. 11(c) and 11(d)], cavity shedding [in Figs. 11(e)-11(g)], and collapse [in Fig. 11(h)] of the entire process. The cavity evolution is approximately axisymmetric, and the average length is smaller than the length on each side in the case near the free surface.

Referring to the aforementioned evolutions, we observe that the cavity on the upper side stays approximately stable and that its length varies in just a small range after the growth stage. The length variation on the lower side is similar to the case under the same cavitation number without the free surface, but has a larger amplitude. These features are linked to the particularities of the current case. For example, the free surface is close to the cavity boundary, and the air above the free surface has
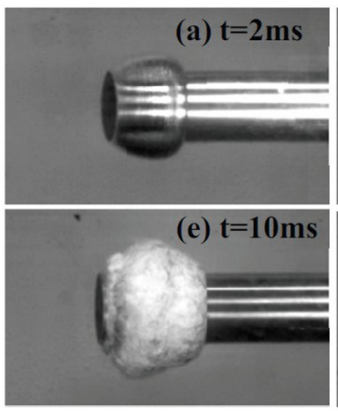
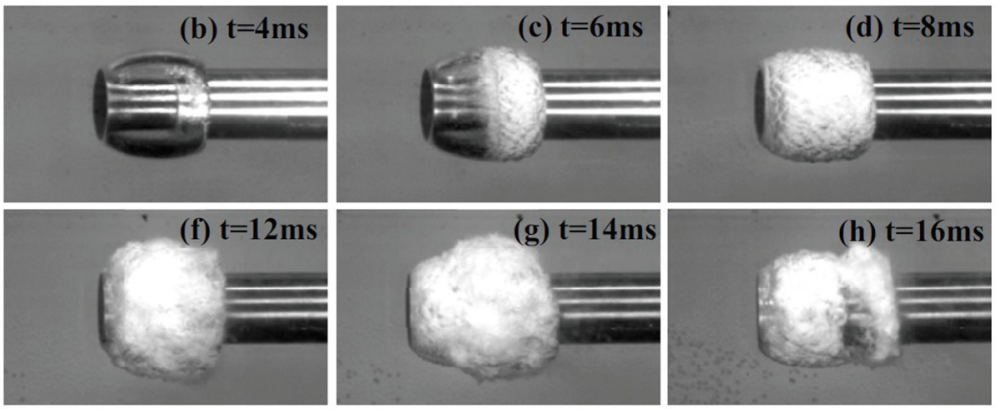

FIG. 11. Time evolution of cavitation patterns obtained from the experiment in the submerged case. The time interval is $2 \mathrm{~mm}$ for each view. 


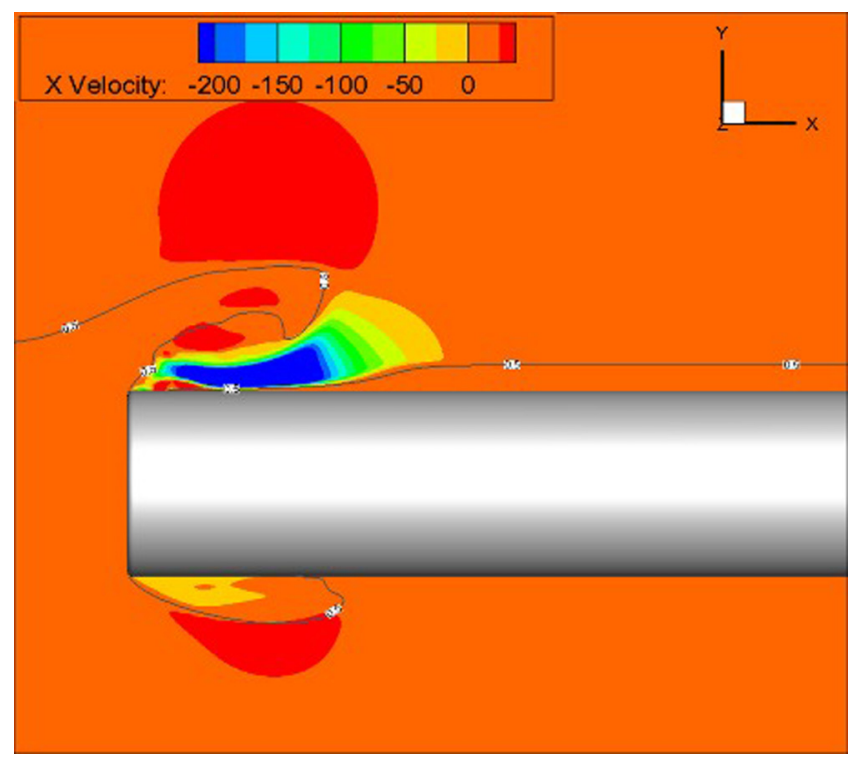

FIG. 12. Flow field of the air entrainment at the beginning (the flow time is equal to $2 \mathrm{~ms}$, and the line represents the interfaces where the volume fraction of the liquid water is equal to 0.5 , while the color represents the velocity in the $X$ direction)

been entrained into the cavity. These characteristics can affect the fluid property inside the cavity and affect the re-entrant jet and other instability factors. These factors will be discussed in detail in subsequent sections.

\section{B. Air entrainment into the cavity}

Passive ventilation occurs in the present case as air entrainment into the cavity, which is a particular feature that may cause the unsteady behavior. The thickness of the cavity increases gradually in the growth stage, and regions of free air space and the cavity will connect after the cavity boundary intersects with the free surface. High-speed air entrainment forms in the connecting channel because the pressure above the free surface is higher than that inside the cavity. At the beginning of air entrainment, the high-speed air flow affects the leading edge of the cavity, which increases the upper cavity boundary and thickens the cavity (Fig. 12). Simultaneously, air flows to the low-pressure region inside the cavity on the lower side and fills it (as shown in Fig. 13). The region influenced by air entrainment is foamlike in the experiment photograph (Fig. 7). Its shape is similar to that of the region with large negative $Y$ velocity in the numerical results (Fig. 13).

Thereafter, the water layer between the cavity and the outside space is affected by the gravity and pressure difference, and moves downward to the wall. The reattachment, which ends the air entrainment, occurs at approximately $4 \mathrm{~ms}$. As shown in Fig. 14, the water layer is curled under the joint effects of gravity and air entrainment, and the air entrainment is compressed just inside a thin gap. The tangential velocity inside the cavity also decreases remarkably at this moment (Fig. 15). The entire air entrainment process lasts approximately $2 \mathrm{~ms}$ in the cavity growth stage, carrying large amounts of air to fill the cavity. On the one hand, air entrainment increases the inner pressure significantly, causing the inner pressure to be higher than the saturation pressure in the natural cavitation bubbles. This difference can be considered as the decrease of the cavitation number inside the cavity. On the other hand, the entrained air is noncondensable, which will remarkably weaken the condensation and collapse processes on the cavity trailing edge and increase the cavity length and stability. 


\section{VENTILATED CLOUD CAVITATING FLOW AROUND A ...}

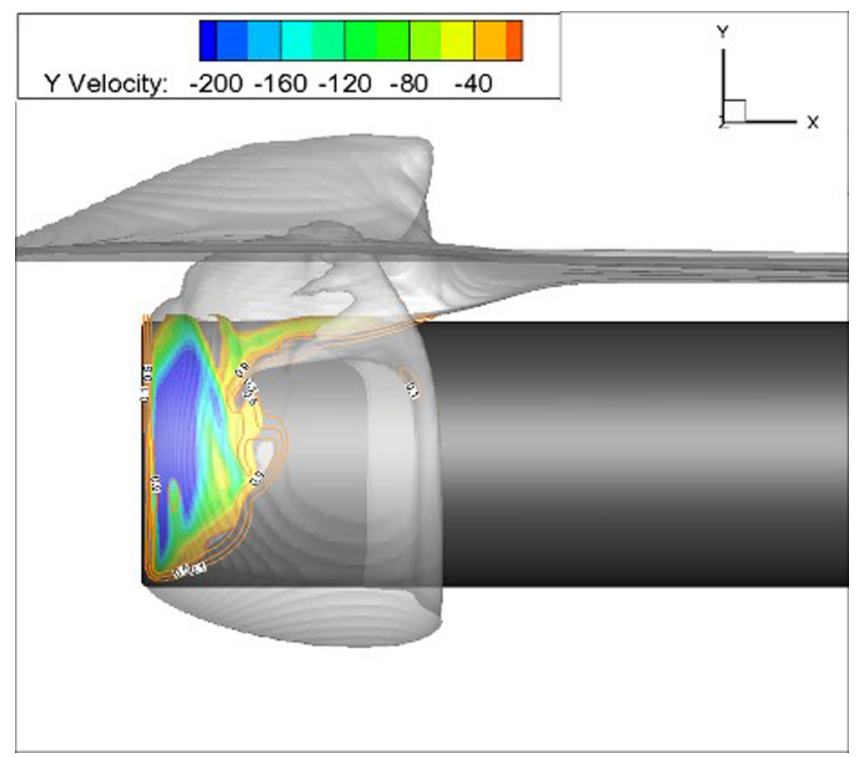

FIG. 13. Distribution of the air entrainment at the beginning (the flow time is equal to $2 \mathrm{~ms}$, translucent surfaces represent the cavity boundary and the free surface, line represents the volume fraction of air, and the color represents the velocity in the $Y$ direction)

\section{The re-entrant jet and cavity shedding}

After the cavity is closed by the water layer, a strong adverse pressure gradient forms in the cavity growth stage between the high-pressure region at the cavity closure and the low-pressure region

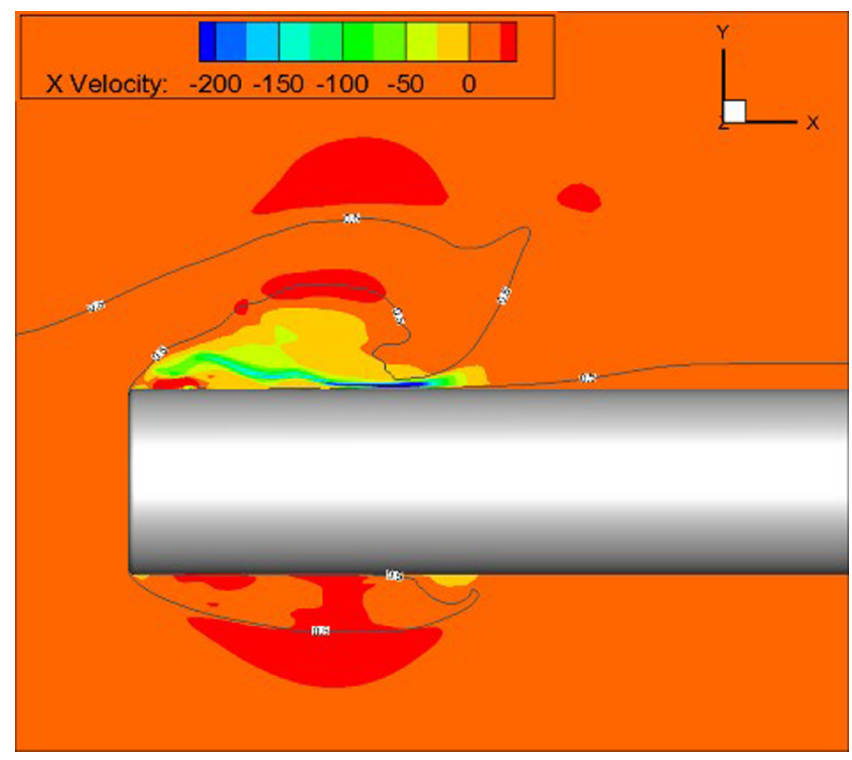

FIG. 14. Flow field of the air entrainment at the end (the flow time is equal to $4 \mathrm{~ms}$, and the line represents the interfaces where the volume fraction of the liquid water is equal to 0.5 , while the color represents the velocity in the $X$ direction). 


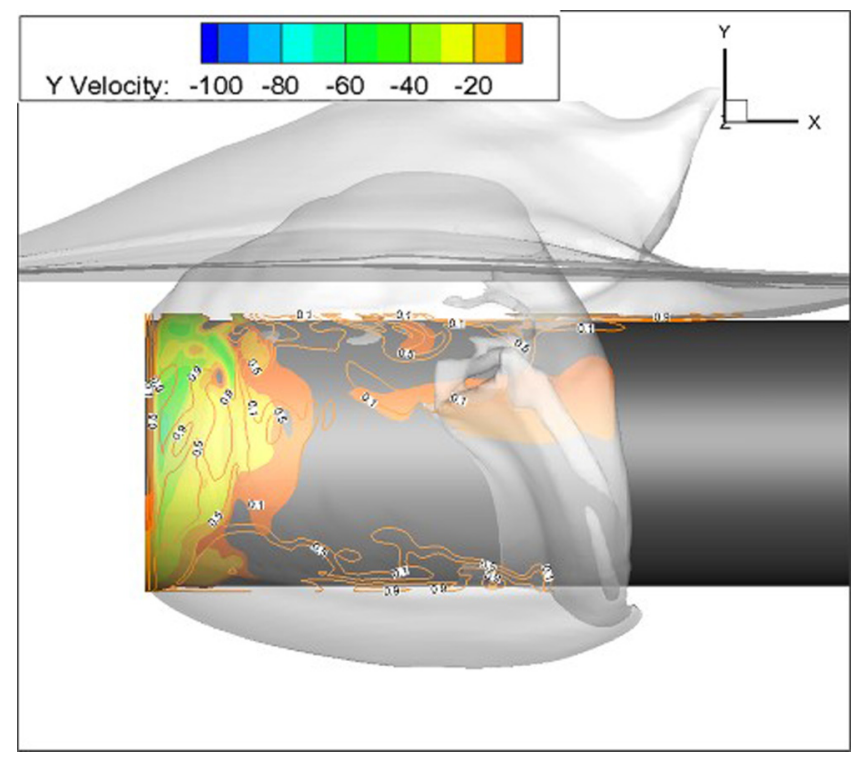

FIG. 15. Distribution of the air entrainment at the end (the flow time is equal to $4 \mathrm{~ms}$, translucent surfaces represent the cavity boundary and the free surface, line represents the volume fraction of air, and the color represents the velocity in the $Y$ direction).

inside the cavity. Flow detachment occurs, and the re-entrant jet is generated as a water layer flows upstream near the wall inside the cavity. An overview of the re-entrant jet shape is as shown in Fig. 16. The water layer of the re-entrant jet is thin on the upper side, whereas it is thicker on the lower side.

The velocity of the re-entrant jet can be observed clearly on the symmetric section plane, as shown in Fig. 17. The re-entrant jet on the upper side of the projectile is thin and flows to the leading edge of the cavity with a high velocity similar to the projectile speed. Theoretically, the thickness of the re-entrant jet is proportional to the thickness of the water layer between the cavity and the free surface, which is also proportional to the submerged depth in the present case. Meanwhile, the water layer starts to flow upstream near the closure of the lower part of the cavity. To see the re-entrant jet more clearly in the figures, the profiles of the re-entrant jet change with time inside the cavity at the upper and lower sides of the projectile are given in Figs. 18 and 19. Specific characteristics of the jet can be observed from the figures. The water layer of the re-entrant jet is thin and flows upstream fast on the upper side, whereas it is much thicker and slower on the lower side. The upturned tail of the re-entrant jet in the upper side cavity can be clearly seen in Fig. 18 which shows the profiles of the re-entrant jet inside the cavity on the upper side of the projectile at $t=5,6,7$, and $8 \mathrm{~ms}$.

The cavity on the upper side is initially cut off with further development of the upper part of the re-entrant jet. However, only a small gap exists between the newly incepted and the remaining cavities because the re-entrant jet is thin (Fig. 20). The gap is soon filled by the growth of the new cavity. By contrast, the re-entrant jet on the lower sides is slower and thicker. A thick front of the re-entrant jet is generated and flows to the leading edge, which can significantly disturb the cavity boundary [as shown in Figs. 20(a)-20(d)]. The cavity boundary is intersected and cut off by the thick front [as shown in Figs. 20(e) and 20(f)]. The experiment photograph (Fig. 8) also shows that the upper part of the cavity is smooth, whereas the middle and lower parts have many small bumps and holes on the surface. When the re-entrant jet reaches the leading edge of the cavity on the lower side, the remaining bubbles only retain a small space of the origin cavity. A region with high liquid water content can separate the new cavity from the remaining part, inducing an obvious shedding 


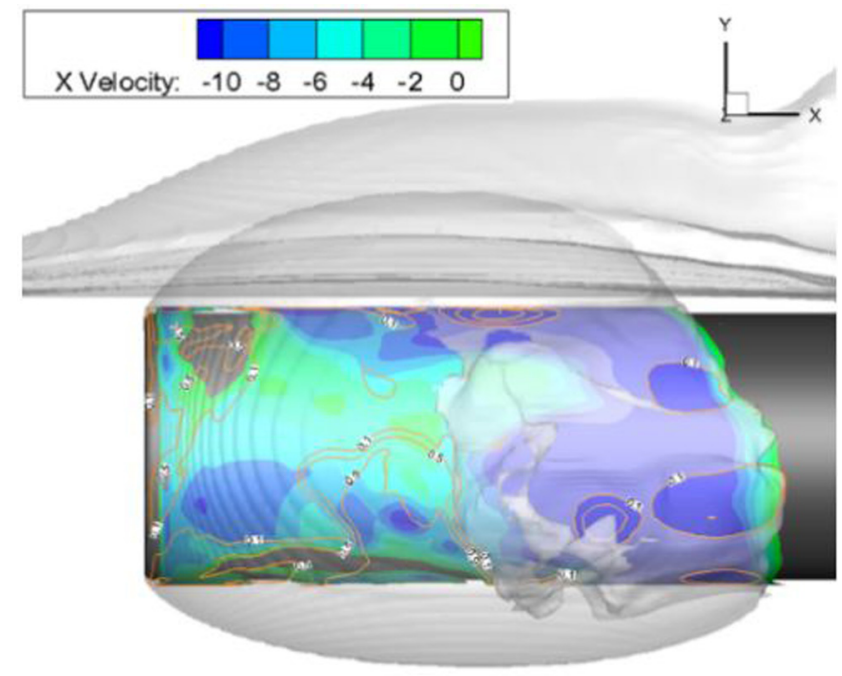

FIG. 16. Shape of the re-entrant jet at the moment of $7 \mathrm{~ms}$. Translucent surfaces represent the cavity boundary and the free surface, line represents the volume fraction of air, and the color represents the velocity in the $X$ direction on the cylindrical surface where the radial coordinate is equal to 1.1 times of radius of the projectile section.

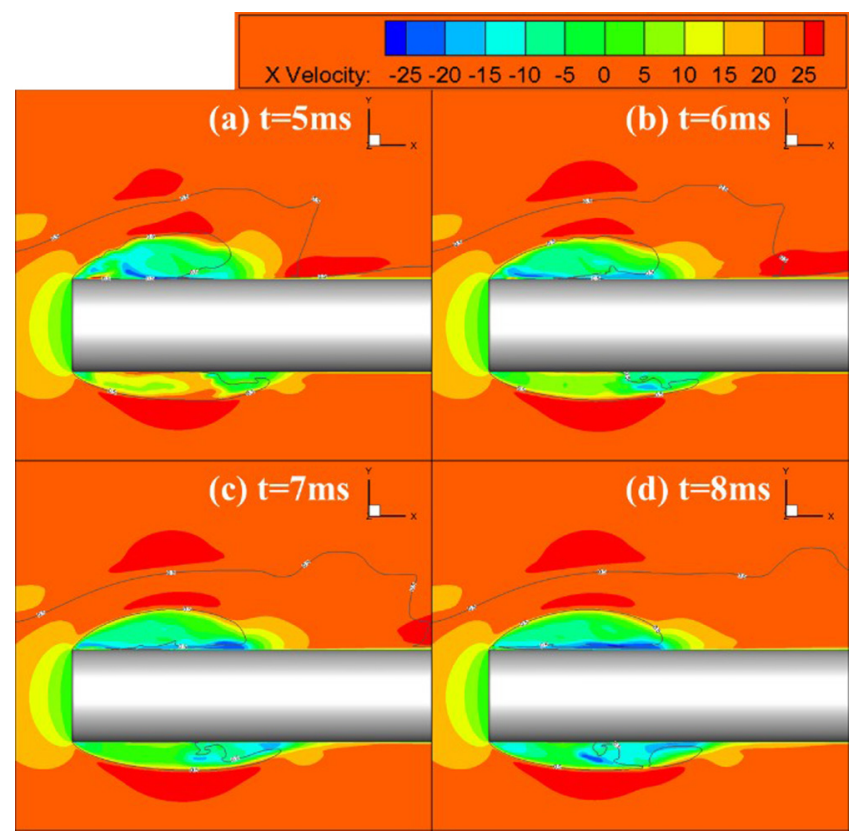

FIG. 17. The development of the re-entrant jet in the upper part of the cavity on the symmetric plane. The line represents the interfaces where the volume fraction of the liquid water is equal to 0.5 , while the color represents the velocity in the $X$ direction. The jet front is pointed out by the white arrow in each view. 


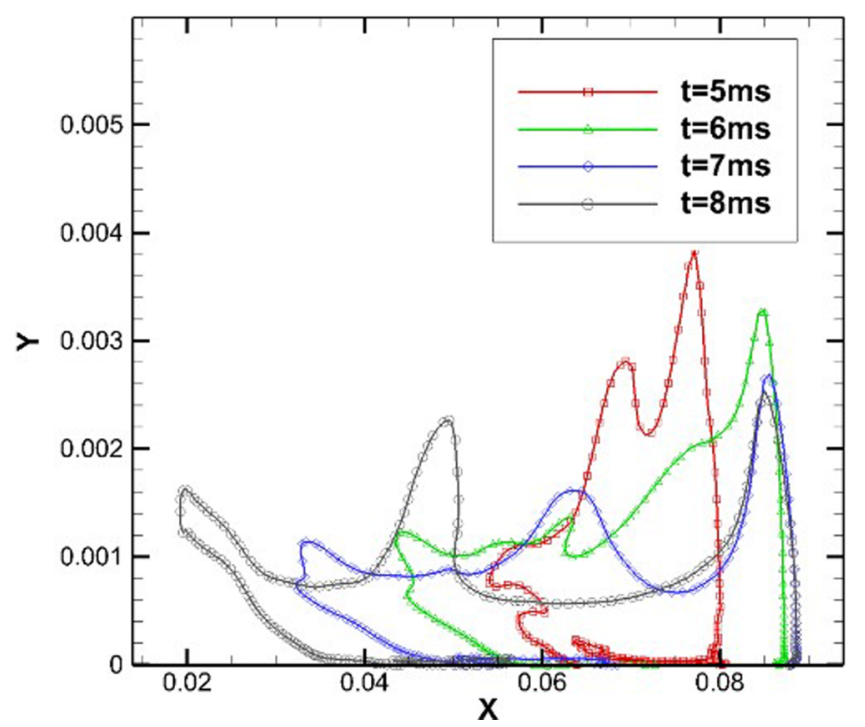

FIG. 18. Profiles of the re-entrant jet inside the cavity on the upper side of the projectile at $t=5,6,7$, and $8 \mathrm{~ms}$.

phenomenon. However, only a small fluctuation occurs on the cavity boundary on the upper side, and the cavity approximately remains in a constant area.

The overview of the re-entrant jet development (Fig. 21) shows that the re-entrant jet has a tangential component from the upper to the lower sides. The pressure is higher at the closure than the lower side because the upper part of the cavity is short and thick. The liquid water accumulates at the re-entrant jet front, which leads to a thick re-entrant jet that can cut off the cavity on the lower side.

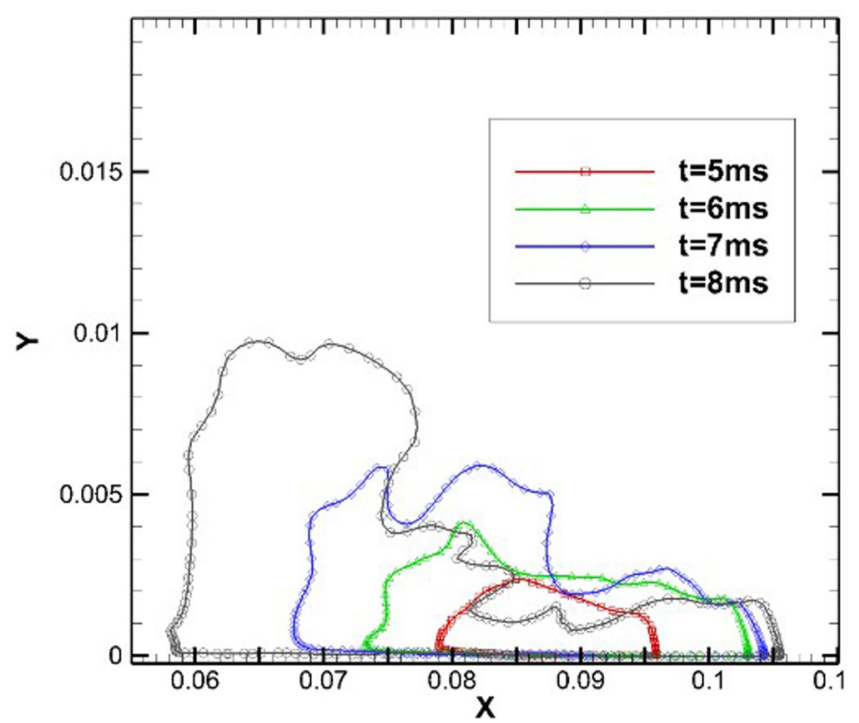

FIG. 19. Profiles of the re-entrant jet inside the cavity on the lower side of the projectile at $t=5,6,7$, and $8 \mathrm{~ms}$. 


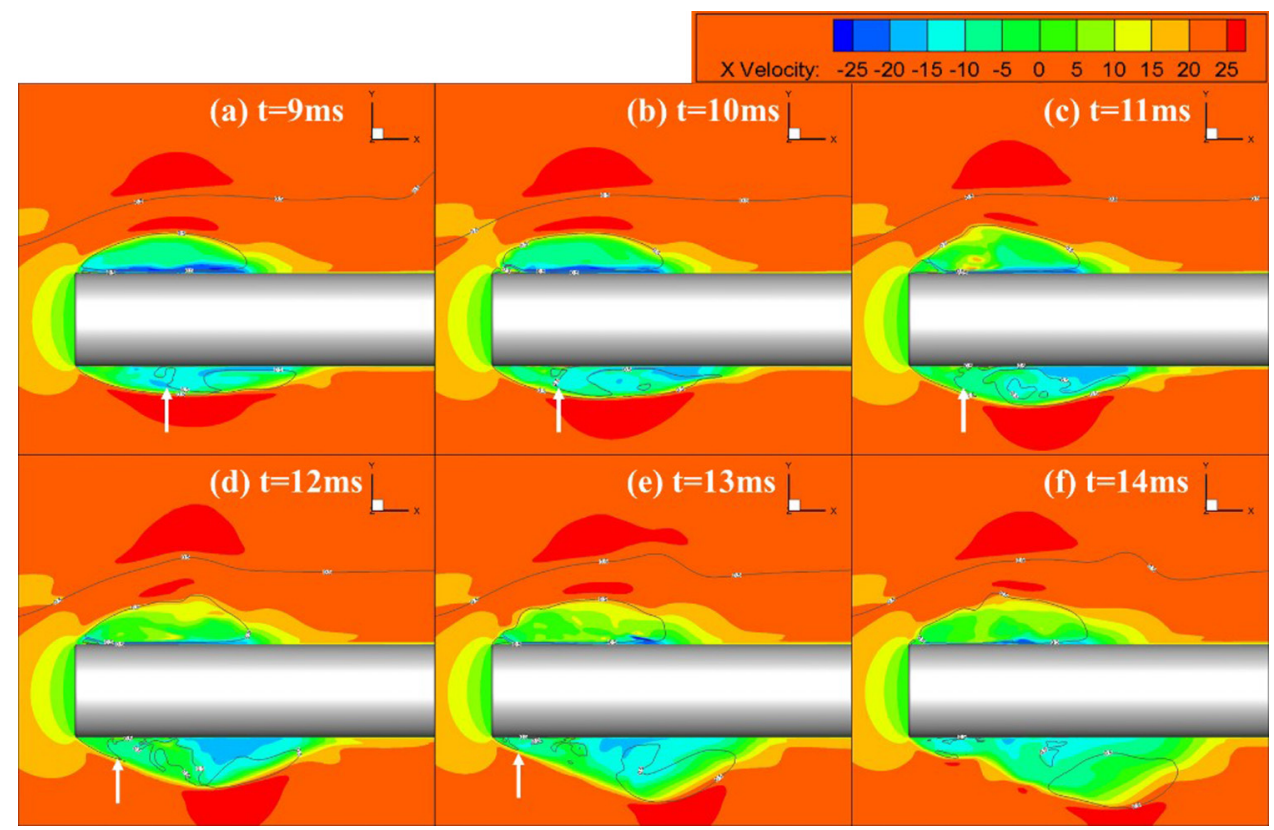

FIG. 20. The development of the re-entrant jet in the lower part of the cavity on the symmetric plane. The line represents the interfaces where the volume fraction of the liquid water is equal to 0.5 , while the color represents the velocity in the $X$ direction. The jet front is pointed out by the white arrow in each view.

The aforementioned results indicate that, if the vehicle moves closely to the free surface, then the water layer flowing around the upper side will be thin and the restriction of the water layer to the flow will be weak. Thus, the generated re-entrant jet will possess insufficient strength and thickness

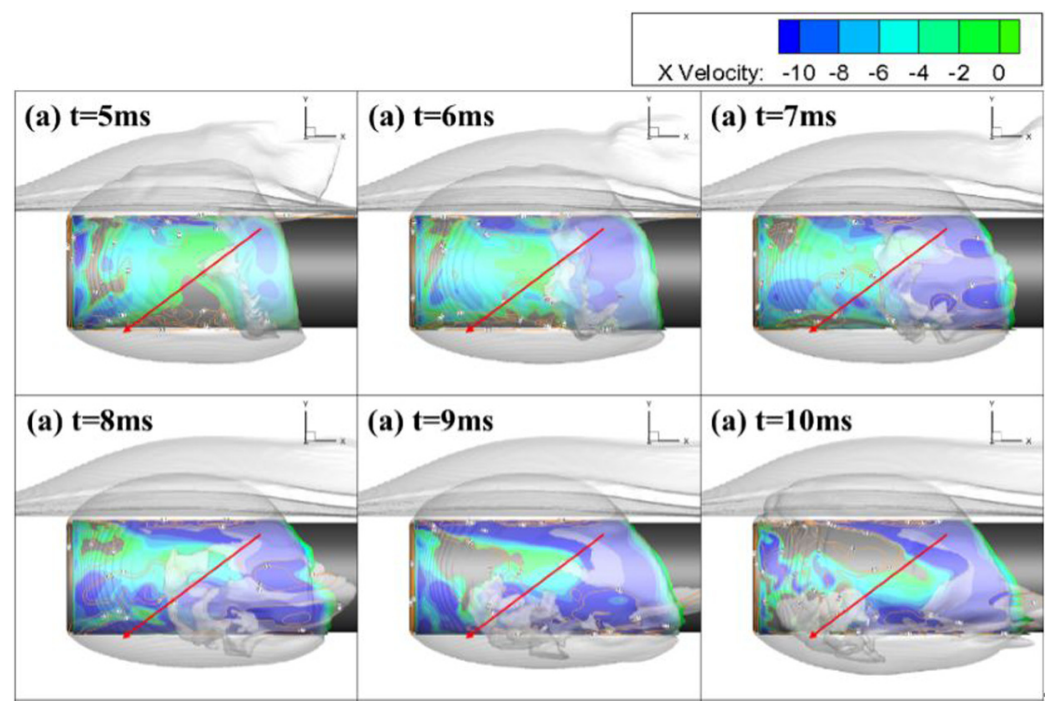

FIG. 21. The development of the re-entry jet inside the cavity on the symmetric plane. The iso-surface represents the interfaces where the volume fraction of the liquid water is equal to 0.5 , while the color represents the velocity in the $X$ direction. The trajectory of the jet front is pointed out by the red arrow in each view. 


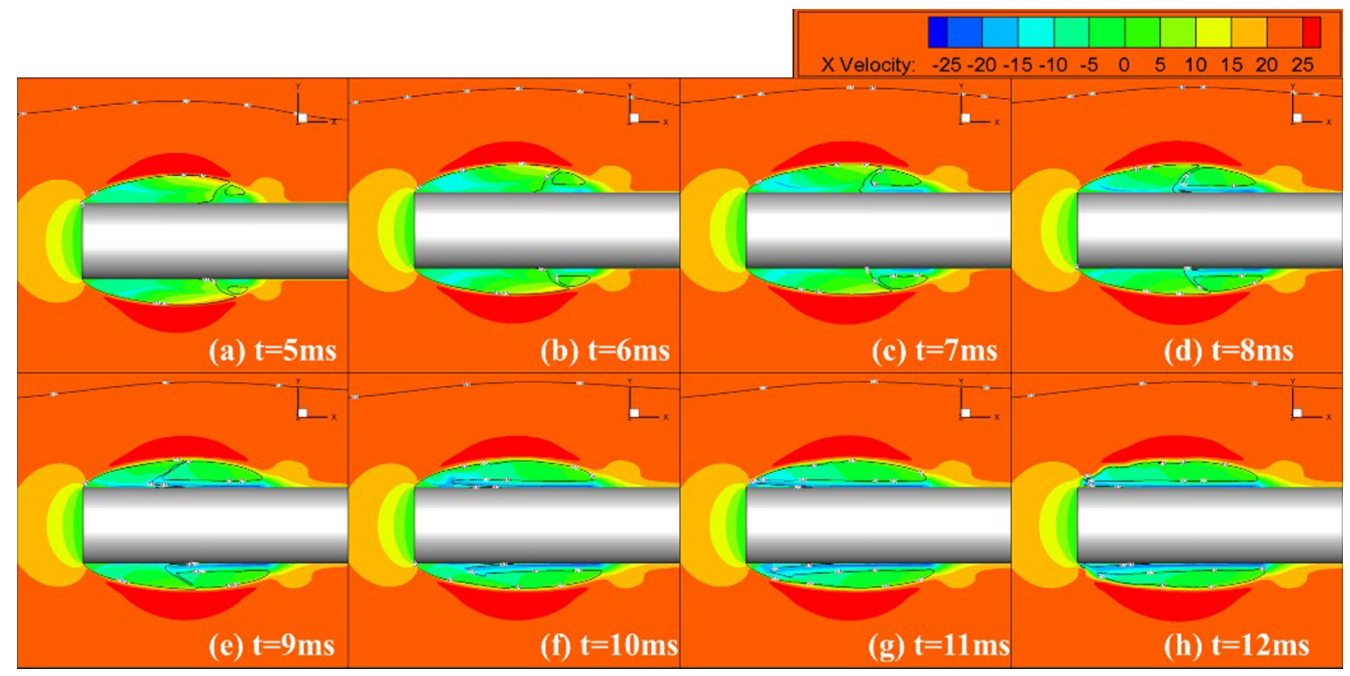

FIG. 22. The development of the re-entrant jet in the cavity on the symmetric plane for the simulated case with $40 \mathrm{~mm}$ distance between the upper side of the projectile and the free surface. The line represents the interfaces where the volume fraction of the liquid water is equal to 0.5 , while the color represents the velocity in the $X$ direction.

to cut off the main cavity completely. Consequently, notable fluctuations of cavity shape will not occur on the upper side. However, the effect of the free surface on the cavity on the lower side is relatively small; thus, a powerful re-entrant jet can be generated, and unsteady evolutions, including breaking and shedding, will still exist.

The re-entrant jet profile for the simulated case with $40 \mathrm{~mm}$ distance between the upper side of the projectile and the free surface is shown in Fig. 22. Comparing to the simulated results when the distance between the upper side of the projectile and the free surface is $5 \mathrm{~mm}$ shown in Figs. 17 and 20, a much thicker re-entrant jet inside the cavity on the upper side of the projectile is shown in the simulated case in deep water, which verified that the free surface nearby could affect the thickness of the re-entrant jet.

\section{Evolution of vortex structures}

The motions of cavity development and shedding are closely linked to vortex structures. The flow structures can be visualized based on the $Q$ criterion. $Q$ has a direct physical interpretation. When $Q>0$, the vector field is dominated by the vorticity and the region is determined as a vortex tube. When $Q<0$, the vector field is dominated by the strain. This physical interpretation supports the value of the $Q$ criterion in the vector fields when areas of high strain and areas of strong vertical motions should be distinguished.

In the present study, the isosurface of $Q=5 \times 10^{6} \mathrm{~s}^{-2}$ represents the vortex core, and the color represents the velocity component in the axial direction. In the air entrainment stage, strong vortices concentrate at the leading edge and air-entrainment channel. The negative value of $X$ velocity is high near the wall in the upper part of the cavity, indicating the existence of intense air entrainment [Fig. 23(a)]. After the channel is closed, most vortices are distributed along the high-speed air entrainment inside the cavity, in which the $X$ velocity is negative on the upper side and positive on the lower side [Fig. 23(b)]. Then, vortices on the upper side weaken and disappear, whereas vortices on the lower side break down into clusters [Fig. 23(c)]. The effect of air entrainment on the velocity field wears off, and vortices are induced by the detachment at the leading edge and the re-entrant jet [as shown in Figs. 23(d) and 23(e)]. Vortex tubes are oblique and normal relative to the aforementioned 


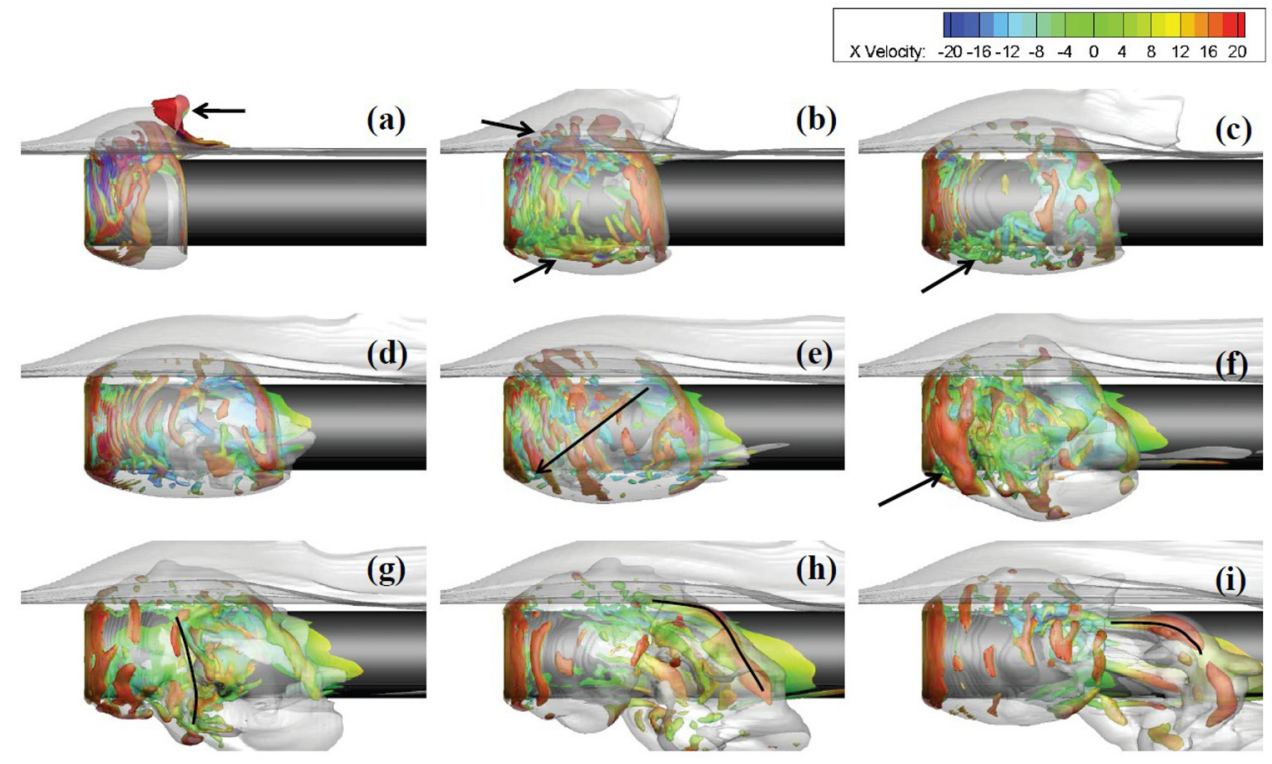

FIG. 23. Vortex structures in the cavity development and shedding process. The isosurfaces represent where $Q=5 \times 10^{6}$, color represents the velocity component in the axial direction, and the outer translucent surfaces represent the cavity boundary and the free surface. Key structures are marked by arrows and lines. The time interval is $2 \mathrm{~ms}$ and the first view is at the moment of $2 \mathrm{~ms}$.

re-entrant jet direction [Fig. 23(e)]. Strong vortices are generated after the re-entrant jet arrives at the leading edge of the cavity [Fig. 23(f)]. Afterward, the cavity is cut off and begins to shed, and vortices form around the gap [as shown in Fig. 23(g)]. Finally, the shedding vortices and cavity begin to break down, and vortex tubes turn parallel to the main flow [as shown in Figs. 23(h) and 23(i)].

The three-dimensional vorticity transport equation is used to analyze the interaction between the vortex and cavitation. The results of the simulated cases with a free surface nearby are compared to the fully submerged case to understand the effect of the free surface on the vortex $[19,28,37]$,

$$
\frac{D \boldsymbol{\omega}}{D t}=(\boldsymbol{\omega} \cdot \nabla) \boldsymbol{V}-\boldsymbol{\omega}(\boldsymbol{\nabla} \cdot \boldsymbol{V})+\frac{\nabla \rho_{m} \times \nabla p}{\rho^{2}{ }_{m}}+\frac{1}{\operatorname{Re}}\left(\nabla^{2} \boldsymbol{\omega}\right) .
$$

The four terms in the equation, namely, vortex stretching, volumetric expansion and contraction (dilatation), baroclinic torque (resulting from the misaligned pressure and density gradients), and viscous diffusion, represent the physical mechanism of the vorticity variation of fluid particles during their motion. Given its much smaller effect on vorticity transport than the other terms for the high Reynolds number flow, the last term in the equation can be ignored during the analysis.

Figures 24-26 present the velocity distribution on the added isosurface when the calculated vortex-stretching, vortex-dilatation, and baroclinic torque magnitudes of the simulated cases are equal to $5 \times 10^{6} \mathrm{~s}^{-2}$ at various times. The cases with and without a free surface show obvious differences. Figure 24 shows that the case with a free surface and air injection into the cavity has a highly complex vortex-stretching magnitude structure. The vortex-dilatation magnitude of the simulated cases in Fig. 25 also shows many disturbances in the case with a free surface. The structure of the case with a free surface is also more broken than that of the case without a free surface. Figure 26 shows that the baroclinic torque magnitude has a small drawn isosurface that does not dominate the results of the vortex around the projectile.

Therefore, the main factor lies in the first term, namely, the vortex-stretching magnitude. Given the small upper constraint under the effect of free surface, the velocity gradient becomes larger and the shedding vortex tends to be stretched toward the final asymmetric profile. The second term, 


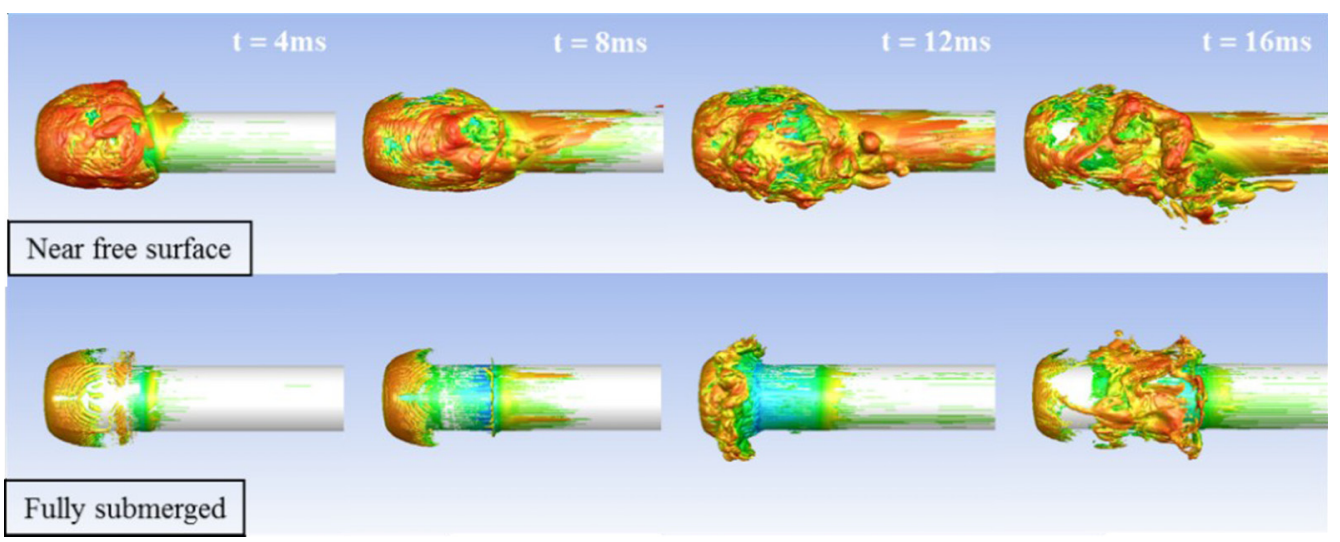

FIG. 24. Velocity distribution on the added isosurface of vortex-stretching magnitude is $5 \times 10^{6}$ at $t=$ $4,8,12$, and $16 \mathrm{~ms}$ of the simulated cases with and without free surface.

vortex-dilatation magnitude, reflects the density change in the continuity equation, which involves the formation and phase transition of the cavity. Therefore, the presented characteristics in Fig. 23 are the same as those of the original form of the cavity. Moreover, when the cavity is generated, the vortex-dilatation magnitude produces a negative effect, thereby weakening the original vortex. The condensed vortex gradually becomes stronger as the generated vortex becomes weaker. The third term, baroclinic torque, reflects the inconsistencies in the direction of pressure and density gradient. This term has a small value because the density change is driven by the pressure change in the flow-dominated phase transition.

\section{CONCLUSIONS}

This paper presented experimental and numerical studies on the ventilated cloud cavitating flow around the axisymmetric projectile near a free surface. The numerical simulation was performed based on the VOF and LES methods. Unsteady behavior, such as air entrainment and shedding of the cloud cavity, was observed, and high consistency was achieved between the numerical and experimental results.

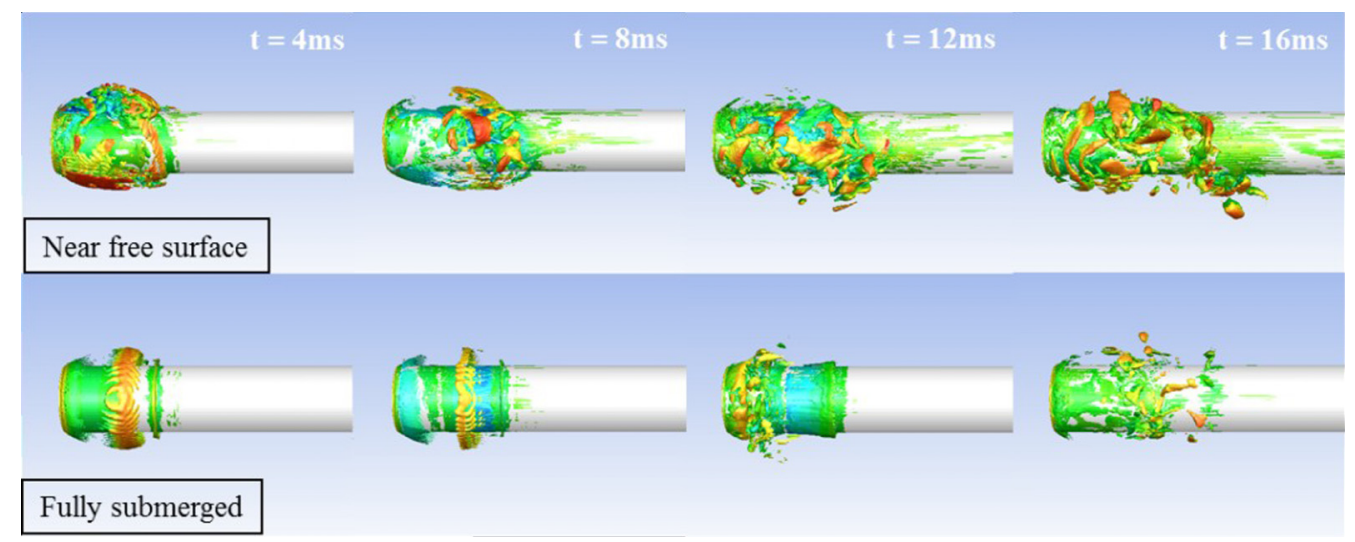

FIG. 25. Velocity distribution on the added isosurface of vortex-dilatation magnitude is $5 \times 10^{6}$ at $t=$ $4,8,12$, and $16 \mathrm{~ms}$ of the simulated cases with and without free surface. 


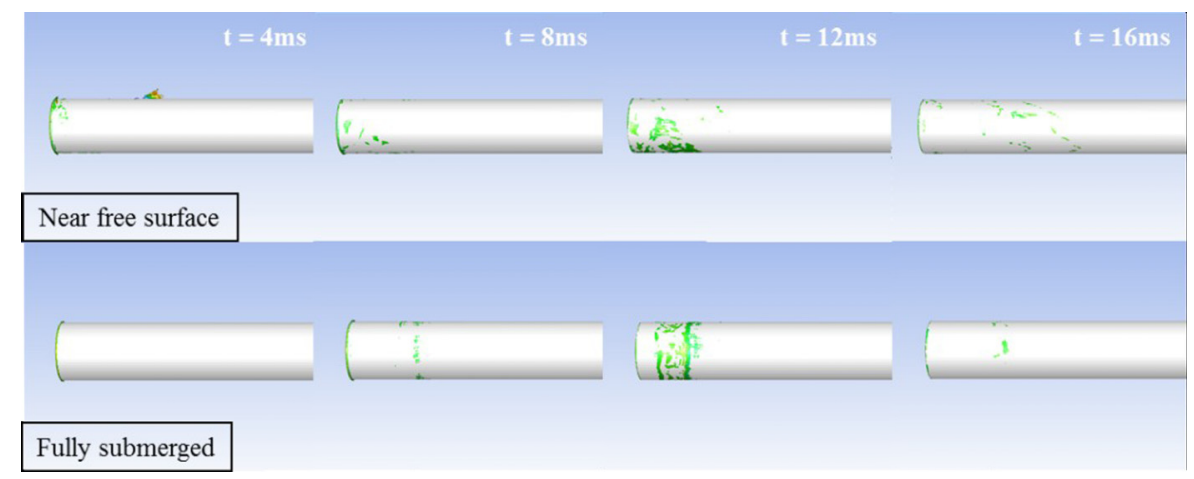

FIG. 26. Velocity distribution on the added isosurface of baroclinic torque magnitude is $5 \times 10^{6}$ at $t=$ $4,8,12$, and $16 \mathrm{~ms}$ of the simulated cases with and without free surface.

Air entrainment occurred when the cavity grew and reached the free surface. The entrained noncondensable air made the cavity larger and more stable than the cloud cavity under a similar cavitation number far from the free surface.

The upper part of the cavity was approximately stable after the growth stage and demonstrated no shedding phenomenon during the whole cavity evolution. This may due to the weak restriction of the water layer to the flow around the upper side of the projectile. The re-entrant jet generated in the upper side cavity was thin and could not cut off the main cavity completely.

The cavity shedding phenomenon on the lower side of the projectile was similar to that of the submerged case. However, the cavity near the free surface was longer. The velocity of the re-entrant jet had a tangential component from the closure of the upper part to the lower side. Strong vortices were mostly generated by air entrainment and the re-entrant jet, and close correlations existed between vortex and shedding cavity motions. The results of the simulated cases with a free surface nearby are compared to the fully submerged case to understand the effect of the free surface on the vortex. Terms in the vorticity transport equation are separately analyzed to identify the dominant physical mechanism. Major differences are observed in the results for vortex stretching and dilatation, thereby suggesting that some disturbances are introduced due to the free surface nearby as well as the air injection into the cavity.

However, several differences regarding the phenomena after cavity shedding existed between the experimental and numerical results, and the current numerical simulation method can be improved in the future. The present study can be extended to vehicles of other shapes including hydrofoils, and the cavity may be kept stable by adjusting the distance between the vehicle and the free surface.

\section{ACKNOWLEDGMENTS}

The authors are grateful for the support of the National Natural Science Foundation of China through Grants No. 11202215 and No. 11332011. This project was also supported by the Youth Innovation Promotion Association CAS (2015015).

[1] O. M. Faltinsen and Y. A. Semenov, The effect of gravity and cavitation on a hydrofoil near the free surface, J. Fluid Mech. 597, 371 (2008).

[2] T. E. Dawson, An experimental investigation of a fully cavitating two-dimensional flat plate hydrofoil near a free surface, California Institute of Technology 11(12), 1651 (1962).

[3] J.-P. Franc and J.-M. Michel, Fundamentals of Cavitation (Springer Science \& Business Media, Berlin, 2006). 
[4] S. Bal and S. Kinnas, A BEM for the prediction of free surface effects on cavitating hydrofoils, Comput. Mech. 28, 260 (2002).

[5] S. Bal, High-speed submerged and surface piercing cavitating hydrofoils, including tandem case, Ocean Eng. 34, 1935 (2007).

[6] S. Bal, The effect of finite depth on 2D and 3D cavitating hydrofoils, J. Marine Sci. Technol. 16, 129 (2011).

[7] E. Amromin, Analysis of body supercavitation in shallow water, Ocean Eng. 34, 1602 (2007).

[8] X. Chen, C. J. Lu, J. Li, and Y. Chen, Properties of natural cavitation flows around a 2-D wedge in shallow water, J. Hydrodyn., Ser. B 23, 730 (2011).

[9] Y. Wang, X. Wu, C. Huang, and X. Wu, Unsteady characteristics of cloud cavitating flow near the free surface around an axisymmetric projectile, Int. J. Multiphase Flow 85, 48 (2016).

[10] B. Stutz and S. Legoupil, X-ray measurements within unsteady cavitation, Exp. Fluids 35, 130 (2003).

[11] B. Stutz and J. L. Reboud, Experiments on unsteady cavitation, Exp. Fluids 22, 191 (1997).

[12] B. Stutz and J. L. Reboud, Measurements within unsteady cavitation, Exp. Fluids 29, 545 (2000).

[13] C. L. Merkle, J. Feng, and P. E. O. Buelow, Computational modeling of the dynamics of sheet cavitation, Third International Symposium on Cavitation (Grenoble, France, 1998), pp. 47-54.

[14] A. K. Singhal, M. M. Athavale, H. Li, and Y. Jiang, Mathematical basis and validation of the full cavitation model, J. Fluids Eng.-Trans. ASME 124, 617 (2002).

[15] R. F. Kunz, D. A. Boger, D. R. Stinebring, T. S. Chyczewski, J. W. Lindau, H. J. Gibeling, S. Venkateswaran, and T. Govindan, A preconditioned Navier-Stokes method for two-phase flows with application to cavitation prediction, Comput. Fluids 29, 849 (2000).

[16] O. Coutier-Delgosha, J. L. Reboud, and Y. Delannoy, Numerical simulation of the unsteady behaviour of cavitating flows, Int. J. Numer. Methods Fluids 42, 527 (2003).

[17] O. Coutier-Delgosha, B. Stutz, A. Vabre, and S. Legoupil, Analysis of cavitating flow structure by experimental and numerical investigations, J. Fluid Mech. 578, 171 (2007).

[18] J. Y. Wu, G. Y. Wang, and W. Shyy, Time-dependent turbulent cavitating flow computations with interfacial transport and filter-based models, Int. J. Numer. Methods Fluids 49, 739 (2005).

[19] B. Ji, X. Luo, R. E. A. Arndt, and Y. Wu, Numerical simulation of three dimensional cavitation shedding dynamics with special emphasis on cavitation-vortex interaction, Ocean Eng. 87, 64 (2014).

[20] B. Huang, G.-y. Wang, and Y. Zhao, Numerical simulation unsteady cloud cavitating flow with a filter-based density correction model, J. Hydrodyn. 26, 26 (2014).

[21] Y. Wang, C. Huang, X. Fang, X. Yu, X. Wu, and T. Du, Cloud cavitating flow over a submerged axisymmetric projectile and comparison between Two-Dimensional RANS and Three-Dimensional Large-Eddy Simulation Methods, J. Fluids Eng-Trans. ASME 138, 061102 (2016).

[22] G. Wang, and M. Ostoja-Starzewski, Large eddy simulation of a sheet/cloud cavitation on a NACA0015 hydrofoil, Appl. Math. Modell. 31, 417 (2007).

[23] R. E. Bensow and G. Bark, Implicit LES predictions of the cavitating flow on a propeller, J. Fluids Eng.-Trans. ASME 132, 041302 (2010).

[24] N. Dittakavi, A. Chunekar, and S. Frankel, Large eddy simulation of turbulent-cavitation interactions in a Venturi nozzle, J. Fluids Eng.-Trans. ASME 132, 121301 (2010).

[25] E. Roohi, A. P. Zahiri, and M. Passandideh-Fard, Numerical simulation of cavitation around a twodimensional hydrofoil using VOF method and LES turbulence model, Appl. Math. Modell. 37, 6469 (2013).

[26] X. Yu, C. Huang, T. Du, L. Liao, X. Wu, Z. Zheng, and Y. Wang, Study of characteristics of cloud cavity around axisymmetric projectile by large eddy simulation, J. Fluids Eng.-Trans. ASME 136, 051303 (2014).

[27] A. Gnanaskandan and K. Mahesh, A numerical method to simulate turbulent cavitating flows, Int. J. Multiphase Flow 70, 22 (2015).

[28] B. Ji, X. Luo, R. E. Arndt, X. Peng, and Y. Wu, Large eddy simulation and theoretical investigations of the transient cavitating vortical flow structure around a NACA66 hydrofoil, Int. J. Multiphase Flow 68 , 121 (2015). 
[29] S. L. Ceccio, Friction drag reduction of external flows with bubble and gas injection, Annu. Rev. Fluid Mech. 42, 183 (2010).

[30] T. M. Pham, F. Larrarte, and D. H. Fruman, Investigation of unsteady sheet cavitation and cloud cavitation mechanisms, J. Fluids Eng. 121, 289 (1999).

[31] Y. Wang, C. Huang, T. Du, X. Wu, X. Fang, N. Liang, and Y. Wei, Shedding phenomenon of ventilated partial cavitation around an underwater projectile, Chin. Phys. Lett. 29, 014601 (2012).

[32] C. Harwood, Y. Young, and S. Ceccio, Ventilated cavities on a surface-piercing hydrofoil at moderate Froude numbers: Cavity formation, elimination, and stability, J. Fluid Mech. 800, 5 (2016).

[33] Y. Young, C. Harwood, F. M. Montero, J. Ward, and S. Ceccio, Ventilation of lifting bodies: Review of the physics and discussion of scaling relations, Appl. Mech. Rev. 69, 010801 (2017).

[34] Y. P. Wei, Y. W. Wang, X. Fang, C. G. Huang, and Z. P. Duan, A scaled underwater launch system accomplished by stress wave propagation technique, Chin. Phys. Lett. 28, 024601 (2011).

[35] P. J. Zwart, G. A. Gerber, and T. Belarmri, A two-phase flow model for predicting cavitation dynamics, Fifth International Conference on Multiphase Flow (Yokohama, Japan, 2004), Vol. 152.

[36] X. Yu, Y. Wang, C. Huang, and T. Du, Study on the influence of phase change rate on cloud cavitation, Proc. Eng. 61, 204 (2013).

[37] B. Ji, X. W. Luo, X. X. Peng, and Y. L. Wu, Three-dimensional large eddy simulation and vorticity analysis of unsteady cavitating flow around a twisted hydrofoil, J. Hydrodyn., Ser. B 25, 510 (2013). 